\title{
Studies on the Influence of Polymeric Additives as Flow Improvers for Waxy Crude Oil
}

\author{
H. I. Al-Shafy ${ }^{1}$, E.A. Ismail ${ }^{2}$ \\ ${ }^{1,2}$ Egyptian Petroleum Research Institute, Petroleum application department, Nasr city, Cairo, Egypt
}

\begin{abstract}
Several techniques have been used to minimize the problems caused by the wax deposition, and the continuous addition of polymeric inhibitors is considered an attractive technological alternative. The copolymer of ethylene acrylic acid, PEAA, was esterified with 1-Docosonol to produce ester copolymer. The produced ester was grafted with vinyl acetate monomer $(30 \%)$ to produce graft ester, PEAA-DcA-g-VA. The products were purified and characterized by FT-IR and ${ }^{1}$ HNMR. The behaviors of crude oils as flow improvers (rheological measurements and pour point depressant) onto Khalda Petroleum Co. (KhPC), Sumpetco and Salam Base crude oils were evaluated. The shear rate-shear stress and shear rate-apparent viscosity were measured at different concentration (from 500 to $3000 \mathrm{ppm}$ ) and temperatures below, equal and above the pour point temperature $\left(15,24\right.$ and $\left.30^{\circ} \mathrm{C}\right)$. A correlation between both results was also obtained. The evaluations of the efficiency of these copolymers as pour point depressants for tested crude oil using 500 to $3000 \mathrm{ppm}$ from the prepared samples were studied. The pour point measurements show good reduction of pour point temperature from 27 to $6{ }^{\circ} \mathrm{C}$ depending on the composition and location of tested crude oil. The results were shown that, the prepared graft copolymers can used as flow improvers.
\end{abstract}

Keywords: - Flow improvers, Oil additives, Pour point depressants, Waxy crudes.

\section{INTRODUCTION}

The paraffin crystals grow as the temperature decreases, creating a crystalline net which begins to trap the molecules of liquid hydrocarbon until the oil cannot flow [1-4]. Paraffin deposition is responsible for the reduction in production, in terms of maintenance and removal of deposits already formed, increasing the cost of producing and transporting oil products and mainly, causing a number of handling problems in regions where the service temperatures are or become seasonally very low [5,6]. Paraffin deposits have caused formation plugging during stimulation treatment and interfered in low temperature oil pumping [7]. Pour point depressants (PPDs)/flow improvers (FIs) are used as chemical additives when transporting crude oils at temperatures below their wax appearance temperatures (WAT). Wax is not the only component in a crude oil. Other constituents in the crude oil i.e. asphaltenes, resins, lighter distillates, polar aromatics etc., should also be considered as important factors while ascertaining the behavior of a crude oil. Asphaltenes are very large heterogeneous molecules with condensed aromatic nuclei, which may associate to form colloidal sized particles that strongly influenced the viscosity of the oil medium and affect the crystallization of the wax [8-11].

There are many kinds of polymers that are used as PPDs to influence the behavior of the paraffin crystallites formation [12- 14]. In order to improve the efficiency of the additives, theoretical analyses explain the interactive mechanism, co-crystallization, nucleation, or improved wax solubility $[15,16]$. The wax deposition inhibitors are polymeric compounds constituted by a hydrocarbon chain which provides the interaction between the additive and paraffin, and a polar segment that is responsible for the wax crystals morphology modification necessary to inhibit the aggregation stage. For this reason, such inhibitors are known as wax crystal modifiers. Several polymers have been evaluated as wax crystal modifiers and poly(ethylene-covinyl acetate) (EVA) copolymers are among the additives that can be used since they present a good efficiency as crude oil flow modifiers and wax deposition inhibitor $[\mathbf{1 7}, \mathbf{1 8}]$. The development of new additives that can solve or minimize such problems is of great interest for the petroleum industry in the whole world. According to Kumar's investigation on pour point depressants [19], an efficient polymeric additive for paraffin oils should be a linear polymer or copolymer that has pendant hydrocarbon chain groups and/or presents hydrocarbon chains in the polymeric backbone. In order to synthesize a polymer that can perform as an agent to reduce the pour point, the following characteristics should be considered: a sufficient number of pendant alkyl groups; alkyl groups with sufficiently long chains; a convenient distance between the hydrocarbon pendant chains; a medium molar mass; in the case of a copolymer, a suitable ratio between the comonomers; a high stability of the additive and, the amorphous or crystalline nature of the additive $[2, \mathbf{5}, \mathbf{6}, \mathbf{2 0}]$.

In this respect, the present work aims to synthesize polymeric grafts based on ethylene acrylic acid copolymer (PEAA). Vinyl acetate monomer was grafted onto PEAA in the presence of bezoyl peroxide. The produced grafts were investigated as PPD and viscosity improvers for Khalda petroleum (KhPC) crude oil. 


\section{EXPERIMENTAL}

\subsection{Materials}

The Poly ethylene acrylic acid (PEAA) west material, 1-Docosanol (DcA), Vinyl acetate, P-Toluene sulfunic acid monohydrate (PTSA), Benzyl peroxide (BP) from Aldrich Chemicals. Two Egyptian waxy crude oils (Sumpetco Mix. and Salam Base Mix.) were used for evaluating the performance of the synthesized polymeric additives. Their physico-chemical characteristics are given in Table (1).

Table 1: Physico-Chemical characteristics of all crude oils were used.

\begin{tabular}{|l|c|c|c|}
\hline \multirow{2}{*}{ Test } & \multirow{2}{*}{ Method } & \multicolumn{2}{c|}{ Crude Oils } \\
\cline { 3 - 4 } & & Sumpetco & Salam Base \\
\hline API Gravity at $60{ }^{\circ} \mathrm{F}$ & ASTMD-1298 & 41.1 & 40.2 \\
\hline Specific Gravity at $60 / 60^{\circ} \mathrm{F}$ & ASTM D-1298 & 0.820 & 0.8241 \\
\hline Wax content, $(\mathrm{Wt} \%)$ & UOP 46/64 & 12 & 8 \\
\hline Asphaltene content, $(\mathrm{Wt} \%)$ & IP 143/84 & 3 & 2.4 \\
\hline Water content, vol. $\%$ & IP 74/70 & 35 & 75 \\
\hline Pour Point, ${ }^{\circ} \mathrm{C}$ & ASTM D-97 & 27 & 24 \\
\hline
\end{tabular}

\subsection{Synthesis of PEAA-DcA Copolymer}

PEAA-DcA copolymer was prepared by reacting PEAA waste with DcA under $\mathrm{N}_{2}$ gas at $140{ }^{\circ} \mathrm{C}$ in oxylene in the presence of $1 \%$ PTSA (wt \% based on total weight of reactants). The reaction was carried out in a four-neck glass flask equipped with a stirrer, thermometer, nitrogen gas inlet and a reflux condenser. The water of the esterification reaction was removed throughout the course of the reaction using Dean and Stark separator. The PEAA copolymer was first dissolved in refluxing o-xylene, and then PTSA catalyst and DcA were added to reaction medium.

\subsection{Grafting of VA onto PEAA-DcA Copolymer}

The esterified PEAA with DcA were placed into reaction reactor with suitable amount of Diesel fuel with concentration $35 \mathrm{wt} \%$. The esterified polymers were blended with $30 \%$ of equivalent weight ratio of vinyl acetate monomer. The mixture was bubbled with nitrogen for $1 \mathrm{~h}$ and the reaction temperature was raised up to $65^{\circ} \mathrm{C}$. Then the initiator BP (with $0.2 \mathrm{wt} \%$ based on total weight of both PEAA and VA monomer) dissolved in toluene with suitable concentration was added to the mixture. The temperature was maintained at the temperature of $65{ }^{\circ} \mathrm{C}$ for $6 \mathrm{~h}$ until the reaction viscosity was increased.

\subsection{Pour Point Measurement}

The tested crude oils $(50 \mathrm{ml})$ were heated up to $65{ }^{\circ} \mathrm{C}$ and the PPD additives were added at different concentration. The solution was shacked for 3 minutes and the temperature was kept constant to 5 minutes according to KhPC procedure. The solution was transferred to bottle test tube in water bath cooled at $48{ }^{\circ} \mathrm{C}$. The tube was transferred to another coling bath cooled down to $24{ }^{\circ} \mathrm{C}$. The tube was transferred to cooling bath cooled down to $0{ }^{\circ} \mathrm{C}$. The pour point temperature was measured at temperature $48{ }^{\circ} \mathrm{C}$, ASTM D 97-93.

\subsection{Rheological Measurements}

A Haake viscometer model Rotovisco RV12 was utilized to measure the dynamic viscosity for untreated and treated crude oil with some selected pour point depressants at different concentrations (from 500 to $3000 \mathrm{ppm}$ ) and at different temperatures above and below pour point of crude oils ranging from 30 to $15^{\circ} \mathrm{C}$. Yield point, and apparent viscosity values were determined.

\subsection{Characterization of the Prepared Grafting Copolymer}

\section{RESULTS \& DISCUSSION}

The present work aims to prepare graft copolymers soluble in petroleum crude oil to be evaluated for improving flow properties of petroleum crude oil. All graft copolymers, have to be purified before characterization.

The purified copolymers were analyzed by FTIR spectroscopy. Spectra of a purified graft copolymer sample are shown in Fig. (1). In this respect, the spectra of PEAA-DcA was selected as representative sample. In this respect, increasing of peak intensity at $1735 \mathrm{~cm}^{-1}$ and decreasing of peak intensity at $1700 \mathrm{~cm}^{-1}$, which represent $\mathrm{C}=\mathrm{O}$ stretching of ester group and carboxylic groups, indicates the conversion of carboxylic acid groups into ester groups. Furthermore, the appearance of strong peak at $1100 \mathrm{~cm}^{-1}$ in all spectra, C-O vibration, indicates the formation of ester graft for PEAA copolymer. On the other hand, the disappearance of the broad peak at 3450-2800 $\mathrm{cm}^{-1}$ (-OH stretching of $\mathrm{COOH}$ group) can be attributed to the formation of ester group for the graft. Copolymer composition was determined by means of IR spectroscopy. To determine the VA contents 
in copolymers having $<20 \mathrm{~mol} \% \mathrm{VA}$, the ratio of absorbance of bands at $610 \mathrm{~cm}^{-1}$ (wagging COOR) and 760 $\mathrm{cm}^{-1}$ (rocking $\mathrm{CH}_{2}$ in Ethylene units) [136-138] was used. To analyses copolymer containing > 20-40 mol\% VA, the absorption bands at $1372 \mathrm{~cm}^{-1}$ (rocking $\mathrm{CH}_{3}$ in acetate groups) and at $2925 \mathrm{~cm}^{-1}$ (stretching $\mathrm{CH}_{3}$ in $\mathrm{E}$ units) [21], were used. The mechanism of grafting based on radical polymerization onto $\mathrm{CH}$ backbone group as illustrated in previous work [22].

In this respect, the chemical structure of PEAA-DcA-g-VA can be confirmed by ${ }^{1} \mathrm{HNMR}$ analysis. The signals in ${ }^{1}$ HNMR spectra of the studied copolymer were assigned according to published data [23]. In this respect, ${ }^{1}$ HNMR spectra of PEAA-DcA-g-VA was selected as representative sample and presented in Fig. (2).

The new signals at 1.97, 4.9-5.3 and $3.8 \mathrm{ppm}$ in spectra of PEAA-DcA-g-VA, which attributed to $\mathrm{COOCH}_{3}, \mathrm{COOCH}_{2} \mathrm{CH}_{3}$ and $\mathrm{CHCO}$ of VA, indicate that VA was grafted onto PEAA chains. On the other hand, the signals at 1.188 and $1.42 \mathrm{ppm}$ are observed in 11 spectra and can be attributed to $\mathrm{CH}_{2}$ of ethylene and VA, respectively. The presence of singlet and triplet signals at $0.812,1.2 \mathrm{ppm}\left(\mathrm{CH}_{3}\right)$ in spectra of PEAA-DcA-g-VA indicates that $\mathrm{COOH}$ group of PEAA were esterified with 1-Docosanol.

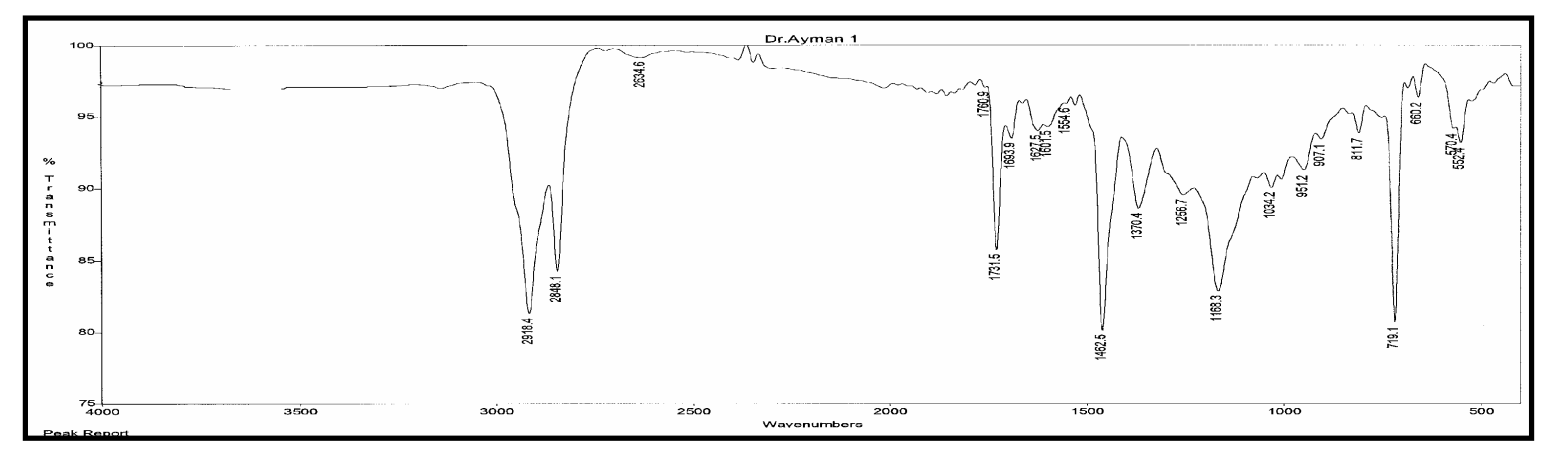

Figure (1): IR Spectrum for PEAA-DcA

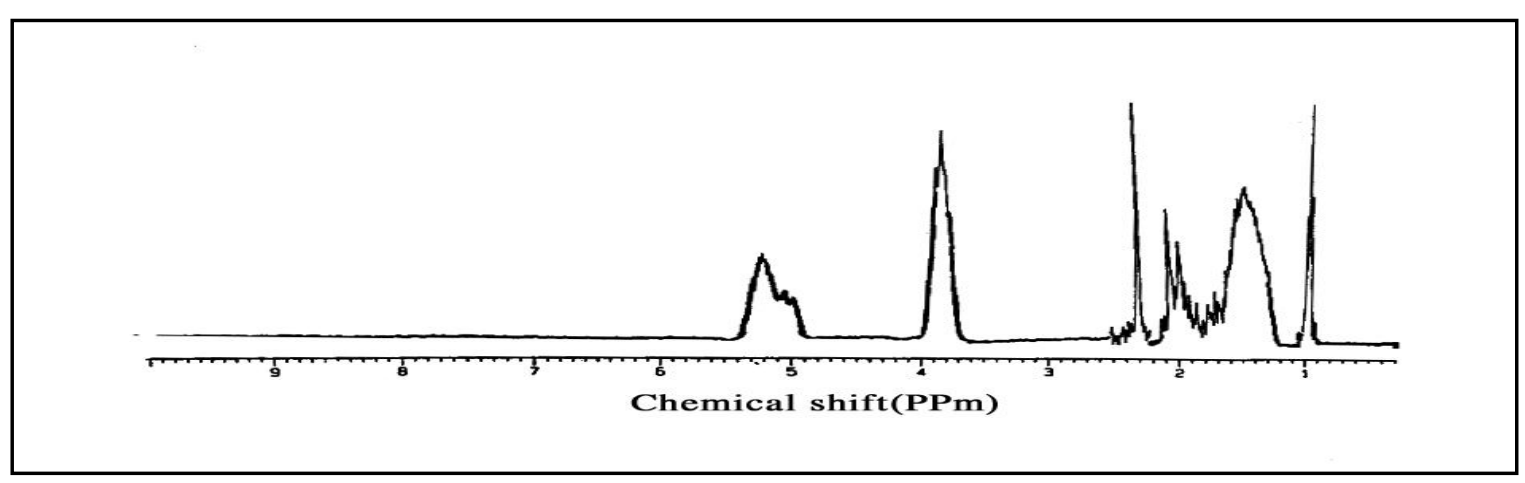

Figure (2): ${ }^{1}$ HNMR Spectrum for PEAA-DcA-g-VA

\subsection{Pour Point Measurements}

Pour points were determined by a modified Amsterdam method. Initially, the crude oil sample was warmed $\left(71^{\circ} \mathrm{C}\right)$ to dissolve the precipitated wax. After this, the sample was left at room temperature to attain 30 ${ }^{\circ} \mathrm{C}$. From this point on, the sample was cooled up to the pour point. Two degrees are added to the temperature, at which the oil sample stopped flowing. The results are presented as pour point reduction in relation to the pour point of the pure crude oil [17]. The pour point reduction was calculated by:

Pour point reduction $(\Delta \mathrm{P})=\mathrm{PP}_{\text {pure }}-\mathrm{P}_{\text {padd }}$

Where, $\mathrm{PP}_{\text {pure }}$ is the pour point of the pure crude oil and $\mathrm{PP}_{\text {add }}$ is the pour point of the crude oil containing additives.

The pour point results and pour point reduction $(\triangle \mathrm{P})$ for pure crude oils and for the crude oil containing additive (PEAA-DcA and PEAA-DcA-g-VA) are shown in Tables $(\mathbf{2}, \mathbf{3})$.

Chemical additives, referred as pour point depressants, flow improvers, paraffin inhibitors or wax crystal modifiers, are widely used for overcoming the problem worldwide. These additives function by one or more several postulated mechanisms, viz. nucleation, adsorption, co-crystallization and improved wax solubility that result in the formation of smaller wax crystals with more regular shape. So the flow improver additives can reduce the growth of the wax crystal and forms smaller crystals to intergrowth and interlock are greatly 
diminished. When the additives or flow improvers are added, they alter the wax crystal size and shape in some manner and prevent the tendency to interlock. The flow improvers or pour point depressants act by regarding the growth of the wax crystals in the XY crystallographic plane, thereby producing smaller crystals have higher volume and surface ratio [24].

The additives were more efficient in reducing the pour point of the oil obtained from the Sumpetco Mix and Salam Base Mix fields from KhPC crude oils. For all the fields, the additives presented the following performance order: PEAA-DcA < PEAA-DcA-g-VA. PEAA-DcA-g-VA achieves the best performance as pour point depressant and viscosity improver at the same concentration of other additives. These behaviors can be correlated to the structure of PEAA grafts that possess 1-Docosanol and vinyl acetate moieties and its interaction with crude oil. However, as vinyl acetate content increases a solubility parameter different from that corresponding to the crude oil, it does not precipitate the sufficient amount that would be necessary to significantly modify the wax crystals [25]. The in Tables $(2, \mathbf{3})$, show the data of untreated and treated crude oils from Sumpetco Mix and Salam Base Mix crude oils. The prepared additives can reduced the pour point temperatures from 27 and 24 to 6 at concentration 3000 ppm onto the crude under investigations.

The relations between $\triangle \mathrm{P}$ and additive concentrations for crude oils without and with additives (PEAADcA and PEAA-DcA-g-VA) are shown in Figs $(\mathbf{3}, \mathbf{4})$. These results depend on concentration of additives and it showing that the $\triangle \mathrm{P}$ value increased with increasing of additive concentrations $500 \mathrm{ppm}$ to $3000 \mathrm{ppm}$ for Sumpetco Mix and Salam Base Mix. On the other hand, the data in the tables and figures indicates that the prepared additives (PEAA-DcA and PEAA-DcA-g-VA) can be used as pure point depressant depending on the composition of crude oils.

Table 2: Pour Point Data of Treated Sumpetco Mix Crude Oil with PEAA-DcA and PEAA-DcA-g-VA

\begin{tabular}{|c|c|c|c|c|c|c|c|c|c|c|c|c|c|}
\hline \multirow{3}{*}{$\begin{array}{c}\text { Crude } \\
\text { oils }\end{array}$} & \multirow{3}{*}{ Dose } & \multicolumn{6}{|c|}{ PEAA-DcA } & \multicolumn{6}{|c|}{ PEAA-DcA-g-VA } \\
\hline & & \multicolumn{2}{|c|}{$10 \%$} & \multicolumn{2}{|c|}{$\mathbf{2 0 \%}$} & \multicolumn{2}{|c|}{$30 \%$} & \multicolumn{2}{|c|}{$10 \%$} & \multicolumn{2}{|c|}{$20 \%$} & \multicolumn{2}{|c|}{$30 \%$} \\
\hline & & $\begin{array}{l}\mathbf{P P} \text {, } \\
{ }^{0} \mathrm{C}\end{array}$ & $\Delta \mathbf{P}$ & $\begin{array}{l}\mathbf{P P} \text {, } \\
{ }^{\circ} \mathrm{C}\end{array}$ & $\Delta \mathbf{P}$ & $\begin{array}{l}\mathbf{P P}, \\
{ }^{\circ} \mathrm{C}\end{array}$ & $\Delta \mathbf{P}$ & $\begin{array}{l}\mathbf{P P}, \\
{ }^{\circ} \mathrm{C}\end{array}$ & $\Delta \mathbf{P}$ & $\begin{array}{l}\mathbf{P P} \text {, } \\
{ }^{\circ} \mathrm{C}\end{array}$ & $\Delta \mathbf{P}$ & $\begin{array}{l}\mathbf{P P}, \\
{ }^{\circ} \mathrm{C}\end{array}$ & $\Delta \mathbf{P}$ \\
\hline \multirow{6}{*}{ 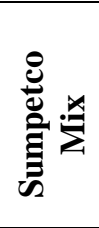 } & Blank & 27 & 0 & 27 & 0 & 27 & 0 & 27 & 0 & 27 & 0 & 27 & 0 \\
\hline & 500 & 27 & 0 & 24 & 3 & 21 & 6 & 24 & 3 & 21 & 6 & 18 & 9 \\
\hline & 1000 & 24 & 3 & 21 & 6 & 18 & 9 & 21 & 6 & 18 & 9 & 15 & 12 \\
\hline & 1500 & 21 & 6 & 18 & 9 & 15 & 12 & 18 & 9 & 15 & 12 & 12 & 15 \\
\hline & 2000 & 18 & 9 & 15 & 12 & 12 & 15 & 15 & 12 & 12 & 15 & 9 & 18 \\
\hline & 3000 & 15 & 12 & 12 & 15 & 9 & 18 & 12 & 15 & 9 & 18 & 6 & 21 \\
\hline
\end{tabular}

Table 3: Pour Point Data of Treated Salam Base Mix Crude Oil with PEAA-DcA and PEAA-DcA-g-VA

\begin{tabular}{|c|c|c|c|c|c|c|c|c|c|c|c|c|c|}
\hline \multirow[b]{3}{*}{$\begin{array}{c}\text { Crude } \\
\text { oils }\end{array}$} & \multirow{3}{*}{ Dose } & \multicolumn{6}{|c|}{ PEAA-DcA } & \multicolumn{6}{|c|}{ PEAA-DcA-g-VA } \\
\hline & & \multicolumn{2}{|c|}{$10 \%$} & \multicolumn{2}{|c|}{$20 \%$} & \multicolumn{2}{|c|}{$30 \%$} & \multicolumn{2}{|c|}{$10 \%$} & \multicolumn{2}{|c|}{$20 \%$} & \multicolumn{2}{|c|}{$30 \%$} \\
\hline & & $\begin{array}{l}\mathbf{P P}, \\
{ }^{\circ} \mathrm{C}\end{array}$ & $\Delta \mathbf{P}$ & $\begin{array}{c}\mathrm{PP}, \\
{ }^{\circ} \mathrm{C}\end{array}$ & $\Delta \mathbf{P}$ & $\begin{array}{c}\mathrm{PP}, \\
{ }^{\circ} \mathrm{C}\end{array}$ & $\Delta \mathbf{P}$ & $\begin{array}{c}\mathbf{P P} \text {, } \\
{ }^{\circ} \mathrm{C}\end{array}$ & $\Delta \mathbf{P}$ & $\begin{array}{c}\mathbf{P P} \text {, } \\
{ }^{\circ} \mathrm{C}\end{array}$ & $\Delta \mathbf{P}$ & $\begin{array}{c}\mathrm{PP}, \\
{ }^{\circ} \mathrm{C}\end{array}$ & $\Delta \mathbf{P}$ \\
\hline \multirow{6}{*}{ 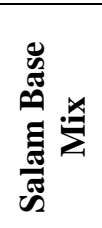 } & Blank & 24 & 0 & 24 & 0 & 24 & 0 & 24 & 0 & 24 & 0 & 24 & 0 \\
\hline & 500 & 24 & 0 & 21 & 3 & 18 & 6 & 21 & 3 & 18 & 6 & 15 & 9 \\
\hline & 1000 & 21 & 3 & 18 & 6 & 15 & 9 & 18 & 6 & 15 & 9 & 12 & 12 \\
\hline & 1500 & 18 & 6 & 15 & 9 & 12 & 12 & 15 & 9 & 12 & 12 & 9 & 15 \\
\hline & 2000 & 15 & 9 & 12 & 12 & 9 & 15 & 12 & 12 & 9 & 15 & 6 & 18 \\
\hline & 3000 & 12 & 12 & 9 & 15 & 6 & 18 & 9 & 15 & 6 & 18 & 6 & 21 \\
\hline
\end{tabular}

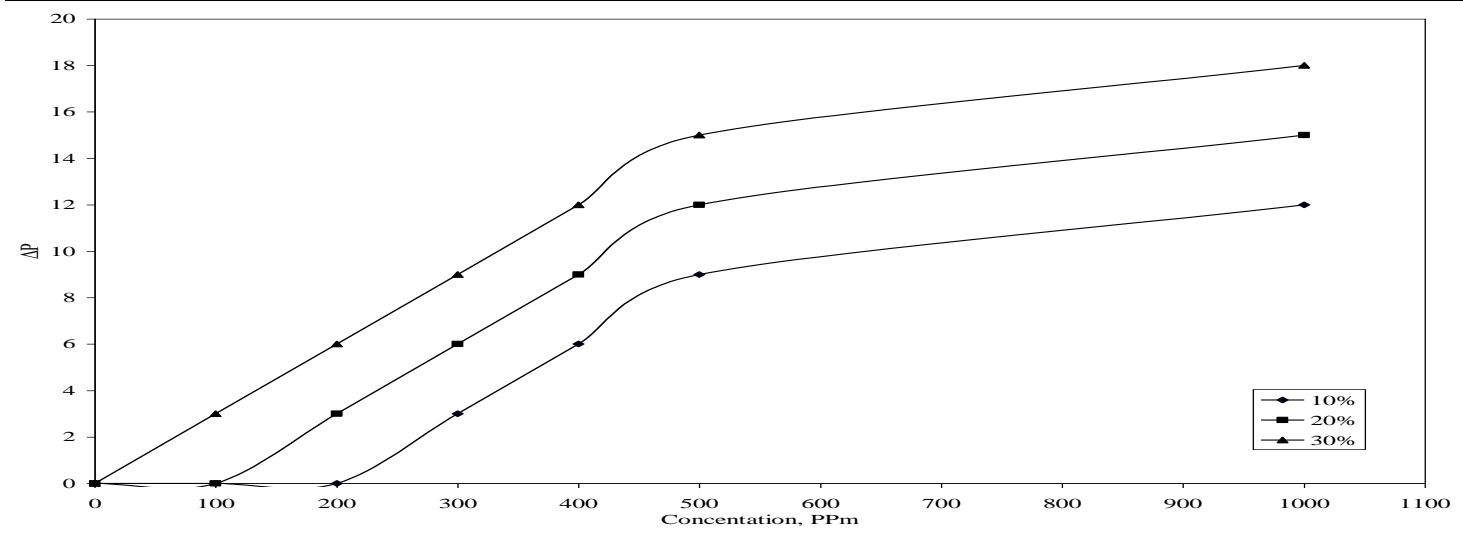

Figure (3): Relation between $\triangle \mathrm{P}$ and concentrations for treated Sumpetco crude oil with $3000 \mathrm{ppm}$ from PEAADcA-g-VA $30 \%$ 


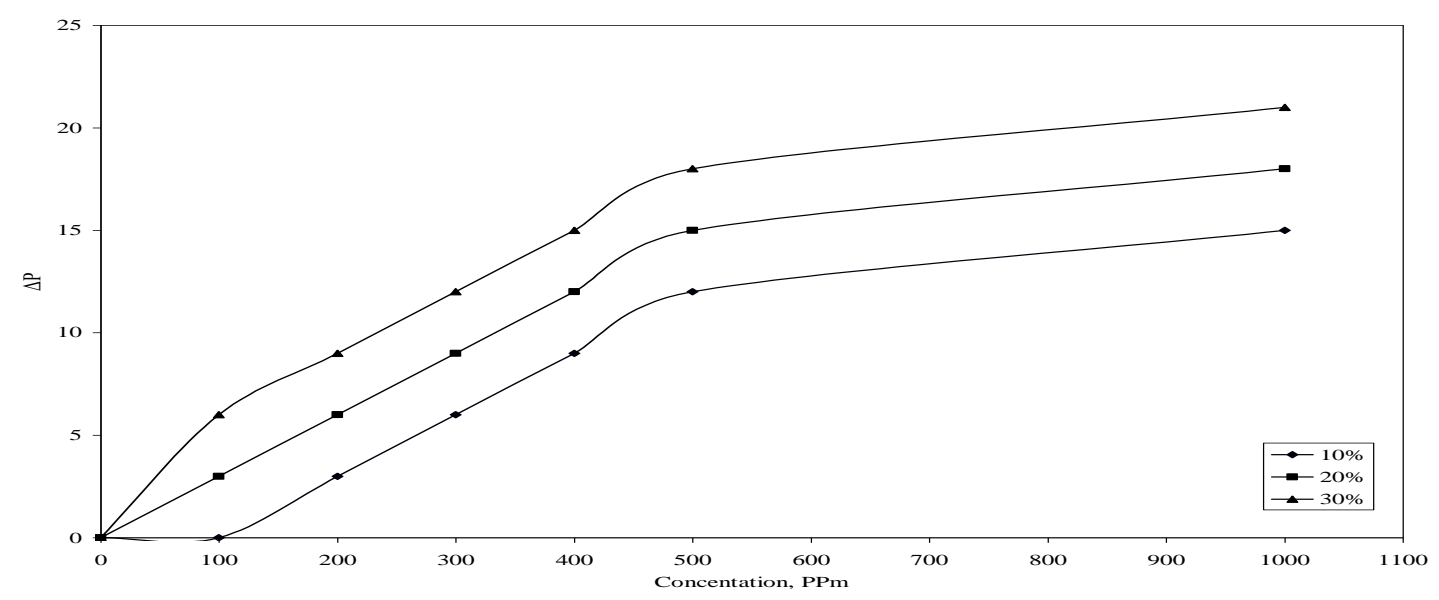

Figure (4): Relation between $\Delta \mathrm{P}$ and concentrations for treated Salam Base Mix crude oil with $3000 \mathrm{ppm}$ from PEAA-DcA-g-VA $30 \%$

\subsection{Rheological Measurements: Evaluation of Graft co-polymers as Flow Improver}

PEAA graft co-polymers were evaluated for their performance as flow improvers for the tested crude oils through rheological measurements at concentration from 500 to $3000 \mathrm{ppm}$. Measurements of the shear rate apparent viscosity and shear rate- shear stress relationships were carried out at different temperatures ranging from $30{ }^{\circ} \mathrm{C}$ to $15{ }^{\circ} \mathrm{C}$. The shear rate - apparent viscosity and shear rate- shear stress relationships for the untreated and treated crude oils at the selected concentration $(3000 \mathrm{ppm})$ of PEAA-DcA-g-VA $30 \%$ are plotted in Figs (5-10) as flow improvers at different temperatures.

It was observed that, the graft co-polymers having alkyl side chains are efficient additives as flow improver. On the other hand, the apparent viscosity - shear rate relationships show non-Newtonian pseudoplastic behaviors at different low concentrations and temperatures (equal or below their pour point) and show Newtonian behaviors at different low concentrations and temperature above their pour point, but their yield stress and plastic viscosity values were decreased as compared with untreated crude oils even at low temperatures. However, the dynamic viscosity decreases with increasing the shear rate reaching a limiting value at high shear rate. This infinite shear rate viscosity is known as the apparent viscosity [10].

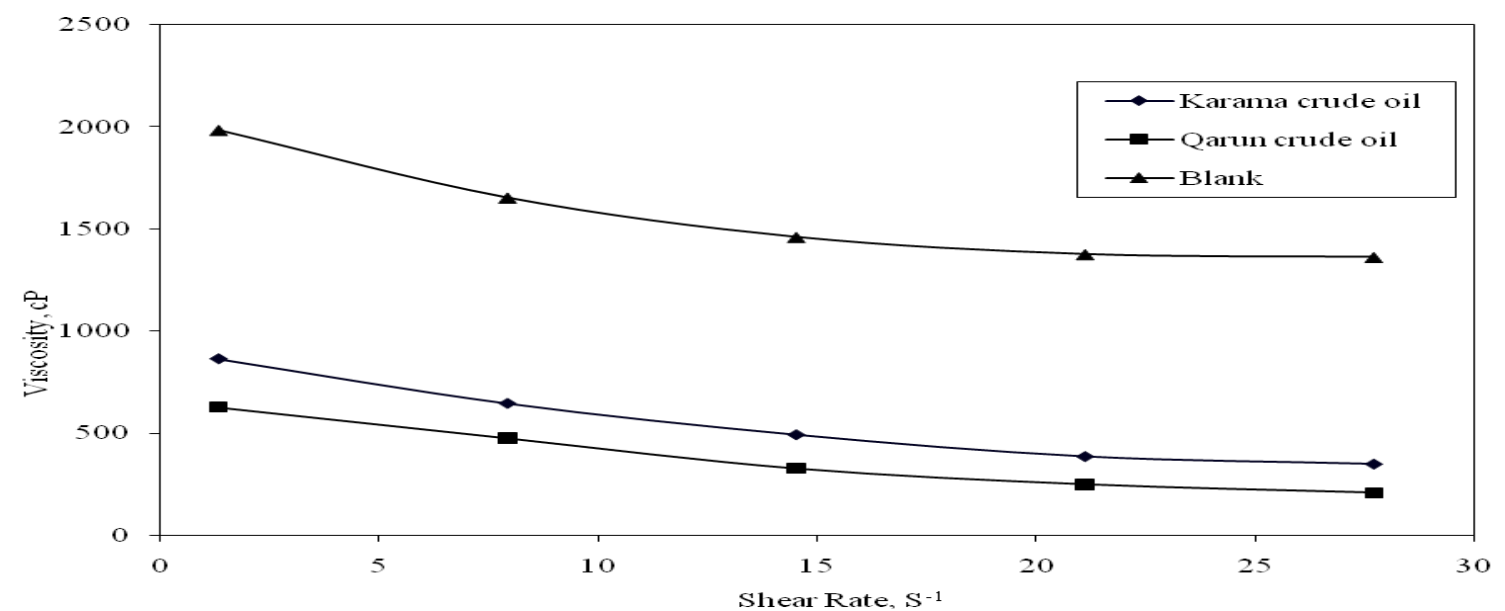

Figure (5): Relation between shear rate and apparent viscosity of KhPC crude oil mix (Sumpetco and Salam Base crude oil) with PEAA-DcA-g-VA $30 \%$ at $3000 \mathrm{ppm}$ and at $15^{\circ} \mathrm{C}$ 


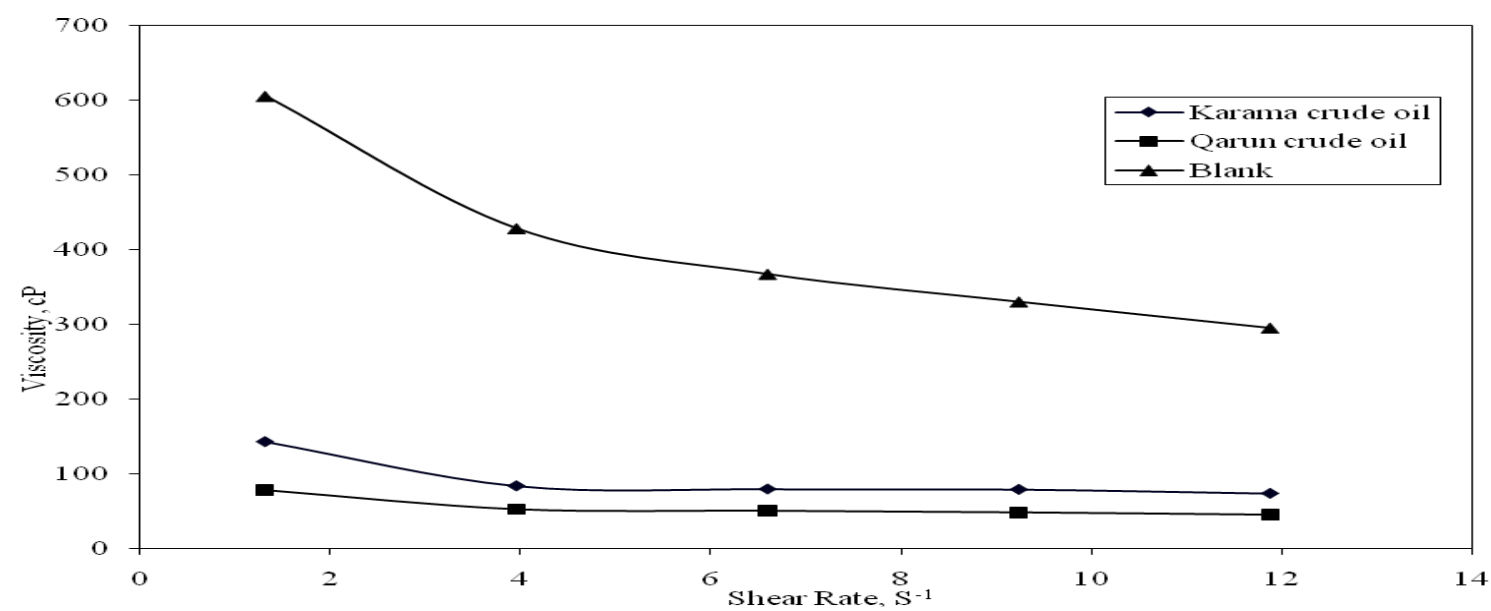

Figure (6): Relation between shear rate and apparent viscosity of KhPC crude oil mix (Sumpetco and Salam Base crude oil) with PEAA-DcA-g-VA $30 \%$ at $3000 \mathrm{ppm}$ and at $24^{\circ} \mathrm{C}$

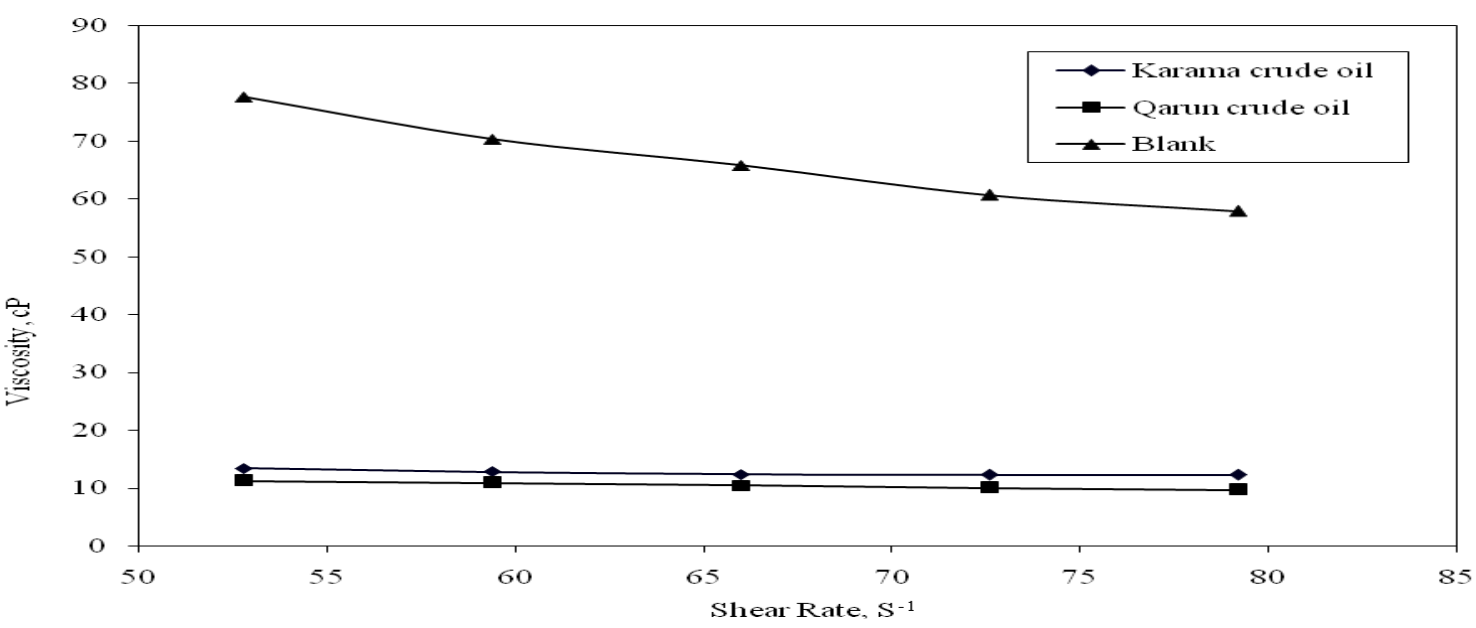

Figure (7): Relation between shear rate and apparent viscosity of KhPC crude oil mix (Sumpetco and Salam Base crude oil) with PEAA-DcA-g-VA $30 \%$ at $3000 \mathrm{ppm}$ and at $30^{\circ} \mathrm{C}$

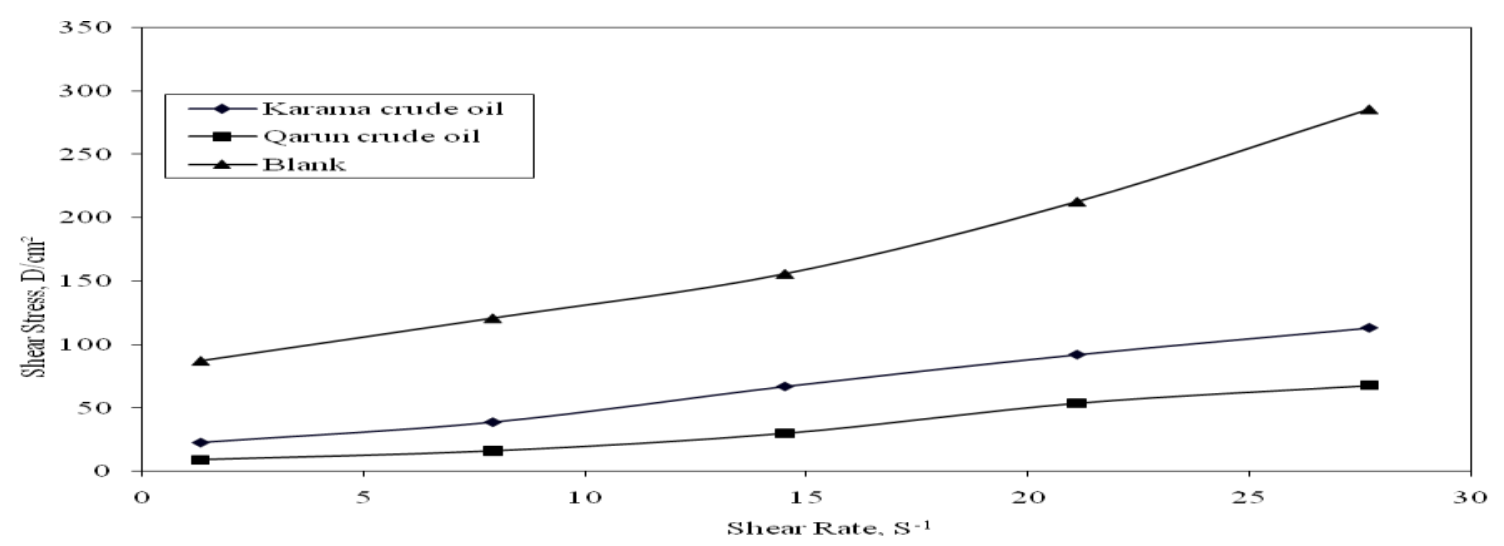

Figure (8): Relation between shear rate and shear stress of KhPC crude oil mix (Sumpetco and Salam Base crude oil) with PEAA-DcA-g-VA $30 \%$ at $3000 \mathrm{ppm}$ and at $15^{\circ} \mathrm{C}$ 


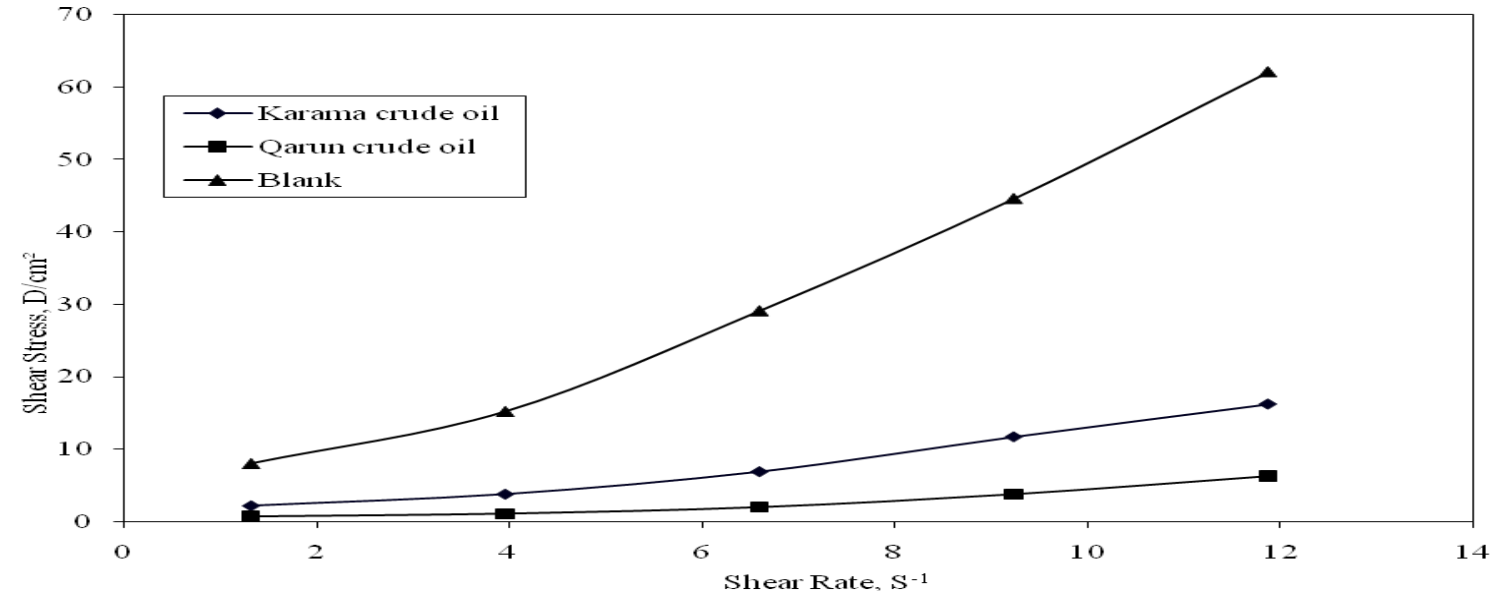

Figure (9): Relation between shear rate and shear stress of KhPC crude oil mix (Sumpetco and Salam Base crude oil) with PEAA-DcA-g-VA $30 \%$ at $3000 \mathrm{ppm}$ and at $24{ }^{\circ} \mathrm{C}$.

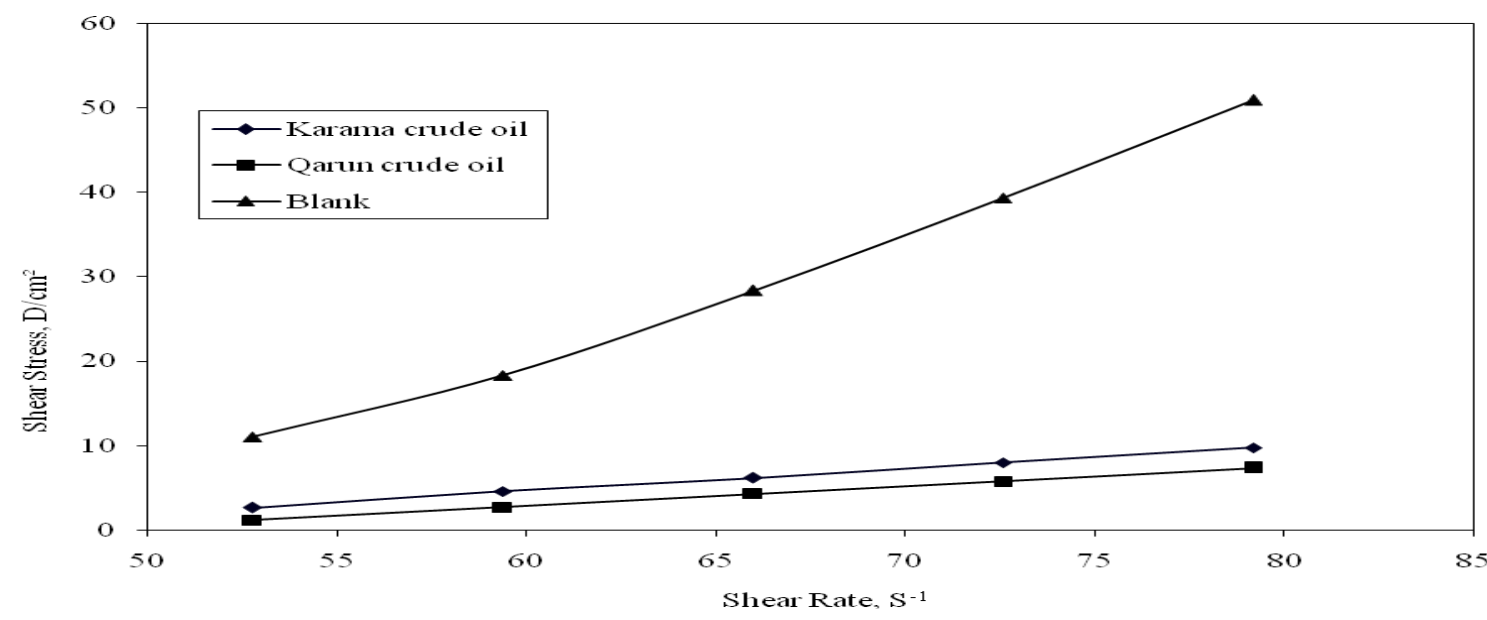

Figure (10): Relation between shear rate and shear stress of KhPC crude oil mix (Sumpetco and Salam Base crude oil) with PEAA-DcA-g-VA $30 \%$ at $3000 \mathrm{ppm}$ and at $30{ }^{\circ} \mathrm{C}$.

These data indicate that graft co-polymeric additives have the ability to disperse asphaltene particles around the wax crystals and improve the flow behavior of the tested crude oils. The behavior of decreasing rheological parameters after addition of the prepared graft co-polymers can thus be attributed to their chemical structure. The high polarity of oxygen in the ester group along the co-polymer chain played a role in preventing the agglomeration of wax crystals in crude oil [18].

\section{CONCLUSION}

1- Prepared of PEAA-DcA-g-VA graft copolymers and evaluation as pour point depressants and rheological measurements. Also, for a given copolymer composition, increasing the size of the alkyl group improves the performance of the additive.

2- Co- polymeric additives obtained in this investigation, a $21{ }^{\circ} \mathrm{C}$ reduction in the pour point of a sample of crude oils, thus demonstrating the efficiency of the products synthesized in this study.

3- For the crude oils of Sumpetco Mix and Salam Base Mix the best performance as pour point depressant was obtained with PEAA- DcA-g-VA.

4- The apparatus was used to test of wax mitigation methods such as pour point depressants for KhPC wax crude oils.

5- The prepared materials show good results as pour point depressant for the tested KhPC crude oils at concentration $3000 \mathrm{ppm}$ for Sumpetco Mix and Salam Base Mix.

6- The data of rheological measurements indicate that the viscometric behaviors of the treated crude oils depend on the crude oil compositions and the structure of the prepared PEAA co-polymeric additives.

7- There is an apparent disagreement between the pour point reduction and the rheological results obtained for the PEAA copolymer. This difference has been ascribed to the different wax particles behavior in the static pour point test and in the measurements involving shearing. 


\section{REFERENCES}

[1] P. Hemant Soni1 and P. Dinakar Bharambe, Synthesis and Evaluation of Polymeric Additives as Flow Improvers for Indian Crude Oil, Iranian Polymer Journal, 15(12), 2006, 943-954.

[2] M.C. Garcia, L. Carbognani, A. Urbina and M. Orea, Paraffin depositon in oil production. Oil composition and paraffin inhibitors activity. J.Petrol. Sci. Technol., 16(9), 1998, 1001.

[3] V. Laura Castro, A. Eugenio Flores and Flavio Vazquez, Terpolymers as Flow Improvers for Mexican Crude Oils, Energy Fuels, 25, 2011, 539-544.

[4] A. Rafael, Soldi, R.S. Angelo, Oliveira, V. Ronilson, Barbosa, A.F. Maria and Ce'sar-Oliveira $J$. European Polymer, 43, 2007, 3671.

[5] Yuping Song, Tianhui Rena, Xisheng Fu, Xiaohong Xu, Study on the relationship between the structure and activities of alkyl methacrylate-maleic anhydride polymers as cold flow improvers in diesel fuels, Fuel Processing Technology, 86, 2005, 641-650.

[6] Z. Huiyang, Z. Weibang and L. Zhuomei, Synthesis of polymers with long side chain of N-alkyl esters and their affects on pour-point depression of oil, J. Appl. Polym. Sci. 43(5), 1991, 919.

[7] K.S. Wang, C.H. Wu, J.L. Creek, P.T. Shuler and V. Tang, J. Pet. Sci. Technol., 21, 2003, 359.

[8] Jun Xu, Huiqin Qian, Shili Xing, $\mathrm{Li} \mathrm{Li}$, and Xuhong Guo, Synthesis of Poly(maleic acid alkylamide-co-rolefin-co-styrene) Co-polymers and Their Effect on the Yield Stress and Morphology of Waxy Gels with Asphaltenes, Energy Fuels, 25, 2011, 573-579.

[9] A. Frohlich and B. Rice, Ind. Crops Prod., 21(1), 2005, 25.

[10] M. Ayman Atta, H. I. Al-Shafey and E.A. Ismail, Influence of Ethylene Acrylic Alkyl Ester Copolymer Wax Dispersants on the Rhological Behavior of Egyptian Crude Oil, J Dispersion Science Technology, 32, 2011, 1296-1305.

[11] A H. I. 1-Shafey, A.I. Hashem, R. S. Abdel Hameed, E. A. Dawood, Studies on the Influence of Long Chain Acrylic Esters Co-Polymers Grafted With Vinyl Acetate as Flow Improver Additives of Crude Oils, Advances in Applied Science Research, 2 (5), 2011, 476-489.

[12] Srushti Deshmukh, D.P. Bharambe, Synthesis of polymeric pour point depressants for Nada crude oil (Gujarat, India) and its impact on oil rheology, Fuel Processing Technology, 89, 2008, 227-233.

[13] K.S. Pedersen, J. Energy Fuels, 17(2), 2003, 321.

[14] Y. Song, T. Ren, X. Fu and X. Xu, J. Fuel Process. Technol., 86, 2005, 641.

[15] J. Zhang, C. Wu, W. Li, Y. Wang and Z. Han,, Fuel, 82(b), 2003, 1919.

[16] A.A. Hafiz and T.T. Khidr, J. Petrol. Sci. and Engin., 56, 2007, 296.

[17] L.C. Andre, A.B. Machado, F. Elizabete, A. Lucas and B. Gaspar Gonza'1ez, J. Petrol. Sci. and Eng., 32, $2001,159$.

[18] H.I. Al-Shafey, R.S. Abdel Hameed, E.A. Ismail, O.E. El Azabawy, Studies of poly ethylene acrylic acid derivatives as pour point depressants of waxy crude oils, Organic Chemistry: An Indian Journal, 10(8), 2014, 308-314.

[19] M.N.S. Kumar, Review on polymeric and copolymeric pour point depressants for waxy crude oils and studies on Bombay high crude oil. Quart J Technol., 15, 1989, 47.

[20] T.V. Fremel, Mecanism of action of pour-point depressants. J. Chem. Tech. Fuels Oil, 29, 1993, 400.

[21] R. J. Koopmans, R. Lihden and E. F. Vansant, Polym. Engng. Sci. 22, 1982, 878.

[22] A. Atta, N. Maysour and K. F. Arndt, J. Polym. Research, 13, 2006, 53.

[23] V. Barboiu, A. Natansohn and D. Blum, Rev. roum. chim. 25, 1980, 933.

[24] L.T. Wardhaugh, and D.V. Boger, Flow characteristics of waxy crude oils, application to pipline design. AIChE J, 37, 1991, 871.

[25] Rafael Martínez-Palou, María de Lourdes Mosqueira, Beatriz Zapata-Rendón, Elizabeth Mar-Juárez, César Bernal-Huicochea, Juan de la Cruz Clavel-López and Jorge Aburto, Transportation of heavy and extra-heavy crude oil by pipeline: A review, Journal of Petroleum Science and Engineering, 75, 2011, 274-282. 